\title{
Tunable two-dimensional electron system at the (110) surface of $\mathrm{SnO}_{2}$
}

\author{
J. Dai, ${ }^{1}$ E. Frantzeskakis, ${ }^{1,2}$ F. Fortuna,,${ }^{1}$ P. Lömker,${ }^{3,4}$ R. Yukawa, ${ }^{5}$ M. Thees,${ }^{1,2}$ S. Sengupta, ${ }^{1}$ P. Le Fèvre, ${ }^{6}$ F. Bertran, ${ }^{6}$ \\ J. E. Rault, ${ }^{6}$ K. Horiba, ${ }^{5}$ M. Müller,${ }^{3,7}$ H. Kumigashira, ${ }^{5,8}$ and A. F. Santander-Syro $\circledast^{1,2,{ }^{*}}$ \\ ${ }^{1}$ Université Paris-Saclay, CNRS, CSNSM-Centre de Sciences Nucléaire et de Sciences de la Matière, 91405, Orsay, France \\ ${ }^{2}$ Université Paris-Saclay, CNRS, Institut des Sciences Moléculaires d'Orsay, 91405, Orsay, France \\ ${ }^{3}$ Peter Grünberg Institut (PGI-6), Forschungszentrum Jülich GmbH, D-52428 Jülich, Germany \\ ${ }^{4}$ Photon Science, Deutsches Elektronen-Synchrotron DESY, Notkestr. 85, 22607 Hamburg, Germany \\ ${ }^{5}$ Photon Factory, Institute of Materials Structure Science, High Energy Accelerator Research Organization (KEK), \\ 1-1 Oho, Tsukuba 305-0801, Japan \\ ${ }^{6}$ Synchrotron SOLEIL, L'Orme des Merisiers, Saint-Aubin-BP48, 91192 Gif-sur-Yvette, France \\ ${ }^{7}$ Fakultät Physik, Technische Universität Dortmund, D-44221 Dortmund, Germany \\ ${ }^{8}$ Institute of Multidisciplinary Research for Advanced Materials (IMRAM), Tohoku University, Sendai 980-8577, Japan
}

(Received 3 March 2019; accepted 24 January 2020; published 14 February 2020)

\begin{abstract}
We report the observation of a two-dimensional electron system (2DES) at the (110) surface of the transparent bulk insulator $\mathrm{SnO}_{2}$ and the tunability of its carrier density by means of temperature or Eu deposition. The 2DES is insensitive to surface reconstructions and, surprisingly, it survives even after exposure to ambient conditions - an extraordinary fact recalling the well known catalytic properties $\mathrm{SnO}_{2}$. Our data show that surface oxygen vacancies are at the origin of such 2DES, providing key information about the long-debated origin of $n$-type conductivity in $\mathrm{SnO}_{2}$, at the basis of a wide range of applications. Furthermore, our study shows that the emergence of a 2DES in a given oxide depends on a delicate interplay between its crystal structure and the orbital character of its conduction band.
\end{abstract}

DOI: 10.1103/PhysRevB.101.085121

\section{INTRODUCTION}

Tin oxide, a transparent insulator, is a technologically important compound with a wide range of applications. Its exceptional properties stem from its ability to exhibit variable oxygen stoichiometries - a consequence of the variable valence of $\mathrm{Sn}$ - accompanied by substantial changes, up to two orders of magnitude, in its conductivity. This remarkable combination of reducibility and changes in conductivity is crucial in the fields of gas sensing [1-8] and heterogeneous catalysis $[3,8-15]$, while the unique association of high conductivity due to intrinsic defects, transparency to visible light, and high resistance to chemical attack at ambient conditions, allow novel applications including solar cells, liquid crystal displays, or even transparent electrodes, conductive coatings, and windows $[5,8,12,16,17]$.

The origin of $n$-type conductivity in $\mathrm{SnO}_{2}$, at the heart of its numerous applications, has thus attracted a large scientific interest. Bulk $\mathrm{SnO}_{2}$ stabilizes in the rutile structure, with a band gap of $3.6 \mathrm{eV}$ [18-21], but the presence of a surface modifies this simple picture. Calculations on the thermodynamically most stable termination, the (110) surface, have proposed $\mathrm{O} 2 p$-derived defect states within the band gap [21]. Early photoemission studies on oxygen deficient $\mathrm{SnO}_{2}$ confirmed the existence of such defect states near the valence band maximum [22-28] and suggested the gradual filling of the band gap upon further reduction $[10,22,29,30]$. This is in

*andres.santander-syro@u-psud.fr contrast with the metallic two-dimensional electron systems (2DESs) observed in similar transparent conducting oxides, such as $\mathrm{In}_{2} \mathrm{O}_{3}$ [31], $\mathrm{CdO}[32], \mathrm{ZnO}[33,34]$, and $\mathrm{TiO}_{2}[35,36]$, where surface defects lead instead to band bending and $n$-type doping of the conduction band.

By means of angle resolved photoemission spectroscopy (ARPES), here we prove the existence of a 2DES at the (110) surface of $\mathrm{SnO}_{2}$ and characterize its electronic structure. Similar to $\mathrm{SrTiO}_{3}$ [37,38] and $\mathrm{KTaO}_{3}$ [39], the 2DES in $\mathrm{SnO}_{2}$ arises from oxygen vacancies and is robust with respect to various surface reconstructions. Intriguingly, the $2 \mathrm{DES}$ in $\mathrm{SnO}_{2}$ is not affected by exposure to ambient air and can be observed without particular surface treatment. Moreover, its carrier density can be tuned by temperature or deposition of $\mathrm{Al}$ or Eu, which create oxygen vacancies at the $\mathrm{SnO}_{2}$ surface, in analogy to previous observations in other oxides [36,40,41]. These results open possibilities of using and controlling the surface conductivity of $\mathrm{SnO}_{2}$ for novel technological applications.

\section{MATERIALS AND METHODS}

\section{A. Crystal structure and Brillouin zone of $\mathrm{SnO}_{2}$}

$\mathrm{SnO}_{2}$ crystallizes in the rutile structure. Similar to the perovskite lattice, the oxygen anions form octahedra around the $\mathrm{Sn}^{4+}$ cation. The primitive unit cell, shown in Fig. 1(a), is body-centered tetragonal. The corresponding 3D Brillouin zone is shown in Fig. 1(b), alongside with its projection on the (110) plane. As will be shown later, the periodicity of 
(a)

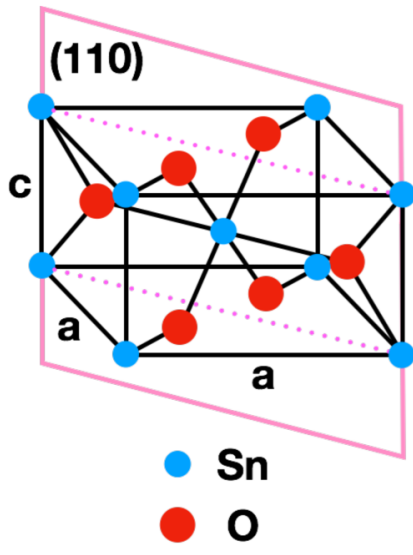

(b)

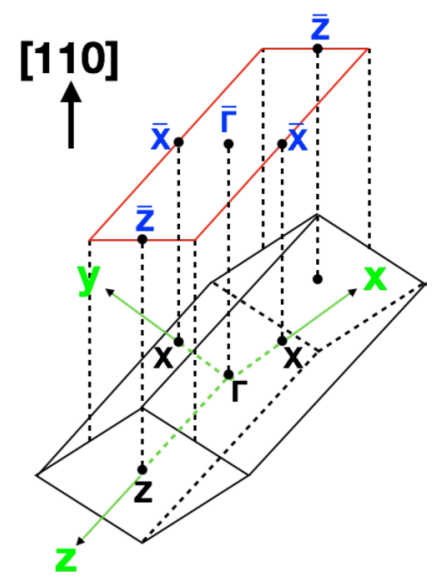

FIG. 1. (a) $3 \mathrm{D}$ conventional unit cell of $\mathrm{SnO}_{2}$ with $a=4.737 \AA$ and $c=3.185 \AA$. (b) Corresponding 3D Brillouin zone of rutile $\mathrm{SnO}_{2}$ (black parallelepiped) together with the surface Brillouin zone projected on the (110) plane (red rectangle).

the shallow metallic state observed in our ARPES data is in perfect agreement with the surface-projected Brillouin zone, confirming the 2D character of such electronic state.

\section{B. ARPES experiments and surface preparation}

ARPES experiments were performed at the CASSIOPEE beamline of Synchrotron SOLEIL (France) and at beamline 2A of KEK-Photon Factory (KEK-PF, Japan) using hemispherical electron analyzers with vertical and horizontal slits, respectively. Typical electron energy and angular resolutions were $15 \mathrm{meV}$ and $0.25^{\circ}$. In order to generate pristine surfaces for the ARPES experiments, commercial (110)-oriented $\mathrm{SnO}_{2}$ single crystals (SurfaceNet) were annealed for 20 minutes at a minimum temperature of $600{ }^{\circ} \mathrm{C}$ in UHV conditions. Low-energy electron diffraction (LEED) and core-level photoemission spectroscopy were employed to verify the longrange crystallinity and cleanliness of our surfaces after preparation. Depending on the annealing temperature, the clean surfaces showed either a $1 \times 1$ bulklike periodicity or a weak $4 \times 1$ surface reconstruction [2,8,12] (see Fig. 2), with no observable difference in the ARPES spectra of the 2DES. As shown as in Fig. 2(c), the energy position and lineshape of photoemission peaks match well with previous experimental results [12].

$\mathrm{Al}$ and Eu deposition were performed by means of molecular beam epitaxy using Knudsen cells, as described in Refs. [36,40]. The deposition rates were calibrated with a quartz microbalance. Unless stated differently, ARPES data shown in this paper were acquired at $T \approx 16 \mathrm{~K}$. The typical pressure during ARPES measurements was in the range of $10^{-11}$ mbar, while at no stage of surface preparation did it exceed $5 \times 10^{-9}$ mbar.

(a)

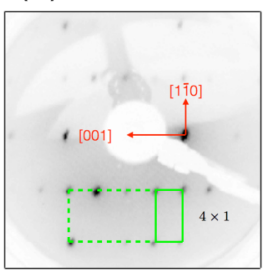

(b)

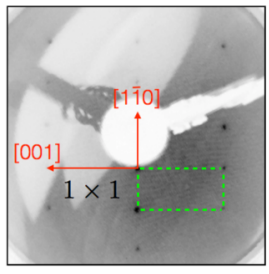

(c)

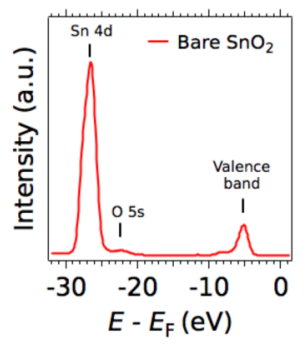

FIG. 2. (a) LEED pattern of the bare (110) surface of $\mathrm{SnO}_{2}$ showing a $4 \times 1$ surface reconstruction after annealing up to $600^{\circ} \mathrm{C}$ in UHV (electron energy: $58 \mathrm{eV}$ ). (b) LEED pattern of the bare (110) surface of $\mathrm{SnO}_{2}$ showing a $1 \times 1$ surface reconstruction after annealing up to $730^{\circ} \mathrm{C}$ in UHV (electron energy: $116 \mathrm{eV}$ ). (c) Angleintegrated spectrum of the $4 \times 1$ reconstructed bare (110) surface of $\mathrm{SnO}_{2}$ showing the profiles of the $\mathrm{Sn} 4 d$ core-level peak and the valence band. The photon energy was $110 \mathrm{eV}$ and the polarization linear horizontal.

\section{RESULTS}

\section{A. Creation of the 2DES}

Figure 3(a), bottom panel, shows the energy-momentum map of the valence band using linear horizontal and linear vertical light polarizations. The observed band dispersions demonstrate the crystallinity and cleanliness of the measured surface, while the strong dependence of the spectra on the light polarization indicates a well-defined orbital character of the corresponding states. Furthermore, in striking contrast with the bulk insulating character of $\mathrm{SnO}_{2}$, here we observe a clear metallic state in the vicinity of the Fermi level $\left(E_{F}\right)$, with a band bottom at an approximate binding energy of $0.2 \mathrm{eV}$-Fig. 3(a), top panel. The energy difference with the valence band maximum (VBM) matches well the energy gap of $\mathrm{SnO}_{2}(3.6 \mathrm{eV})$ as determined by previous experimental and theoretical studies [18-21]. We therefore attribute the metallic state to the conduction band of $\mathrm{SnO}_{2}$ that has been pulled below $E_{F}$ due to band bending, as observed in surfaces of other bulk insulating oxides [33,35-38,41-48]. However, in contrast to the $d$-orbital character of the conduction band in transition metal oxides, the conduction band bottom in $\mathrm{SnO}_{2}$ originates mainly from the $5 s$ states of Sn [20].

Figure 3(b) presents a zoom over the metallic state with higher energy resolution. The dispersion is quasiparabolic, with a kink at an energy of about $80 \mathrm{meV}$ (red arrows) and an intensity buildup at the bottom of the band. Such a kink and strong renormalization of the band bottom are characteristic signatures of electron-phonon interaction, as previously observed in other oxides such as $\mathrm{SrTiO}_{3}[42,49,50], \mathrm{TiO}_{2}$ [36], and $\mathrm{ZnO}$ [33]. The Fermi momentum $\left(k_{F}\right)$ of the metallic state, determined from the maxima of the momentum distribution curve (MDC) integrated over $E_{F} \pm 2 \mathrm{meV}$, is $k_{F}=$ $(0.077 \pm 0.001) \AA^{-1}$.

Figure 3(c) shows the in-plane ARPES intensity map at $E_{F}$ measured over several neighboring Brillouin zones. The aforementioned metallic state gives rise to a circular Fermi contour around the center of, and with the same reciprocalspace periodicity as, the surface-projected Brillouin zone of 

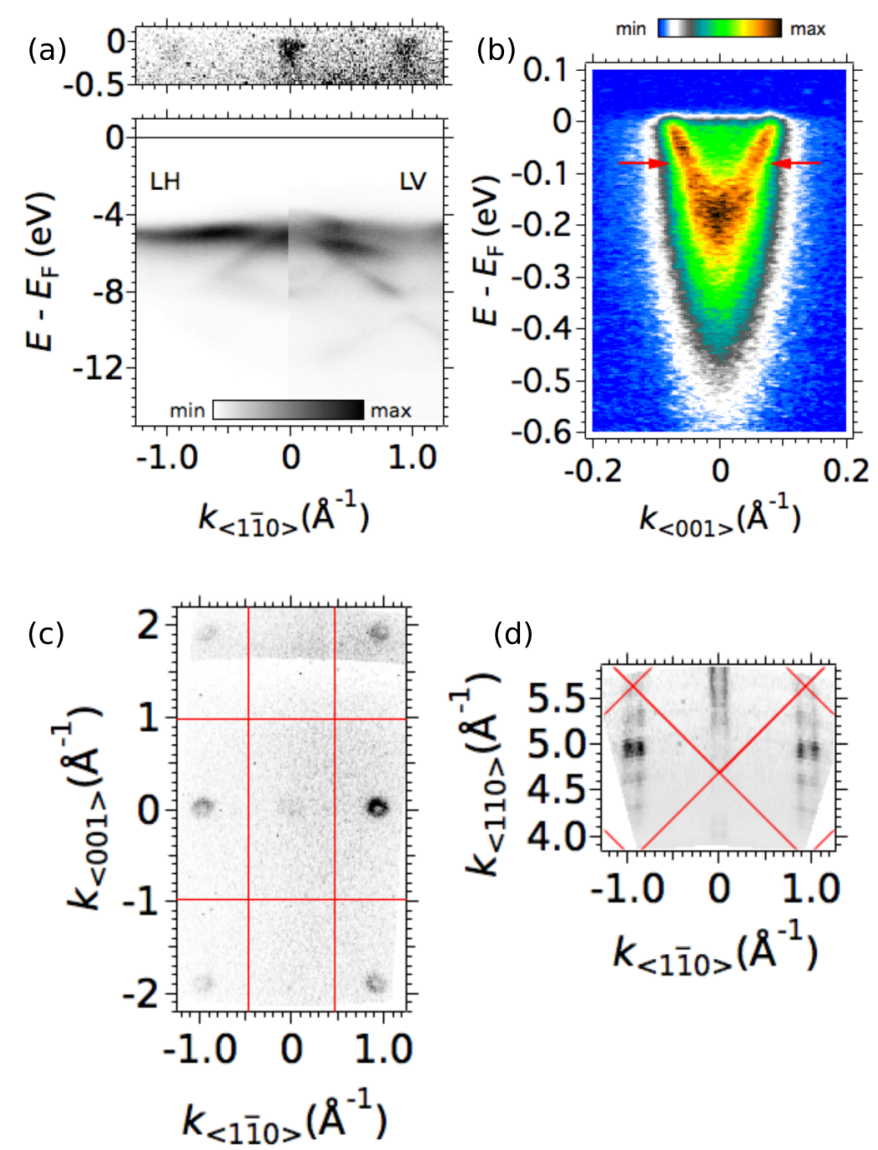

(d)

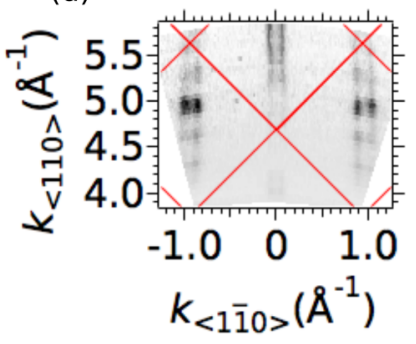

FIG. 3. (a) ARPES energy-momentum map of the valence band on the bare (110) surface of $\mathrm{SnO}_{2}$ measured with photons of energy $111 \mathrm{eV}$ using linear horizontal (LH, left part) and linear vertical (LV, right part) polarizations. The top panel shows a shallow metallic state, fingerprint of a 2DES, made visible after changing the contrast of the color scale. (b) Zoom over the energy-momentum dispersion of the 2DES $(h v=88 \mathrm{eV})$. Electron-phonon interaction induces the observed deviations (red arrows) from a simple parabolic shape. (c) In-plane Fermi surface map of $\mathrm{SnO}_{2}$ (110) measured with $\mathrm{LH}$ photons at $h v=88 \mathrm{eV}$. (d) Out-of-plane Fermi surface map of $\mathrm{SnO}_{2}(110)$ acquired by a stepwise change of the photon energy between 50 and $120 \mathrm{eV}$ using LH polarization. An inner potential of $10 \mathrm{eV}$ was used in the calculation of the out-of-plane momentum. The blue dashed line indicates the $h v=88 \mathrm{eV}$ constant energy line, where the in-plane Fermi surface in (c) was measured. Red lines mark the borders of the projected surface Brillouin zone in (c) and of the bulk Brillouin zones in (d). All data in this figure were acquired at $16 \mathrm{~K}$.

the bulk rutile structure of $\mathrm{SnO}_{2}$ shown in Fig. 1(b). Moreover, the isotropic shape of the Fermi contours, and especially the strong dependence of their ARPES intensity on different Brillouin zones and/or different photon polarizations [51] (Appendix A), are in line with the expected $s$-like orbital character at the bottom of the $\mathrm{SnO}_{2}$ conduction band, as discussed above. We note that the 2DES is insensitive to the $4 \times 1$ surface reconstruction observed in some of our in situ prepared surfaces, Fig. 2(a), as no Umklapp band structure is observed by ARPES. This suggests that the 2DES resides in the subsurface region (1-2 unit cells below the surface as analyzed later), in agreement with previous conclusions on the (111)-oriented surfaces of $\mathrm{SrTiO}_{3}$ [38] and $\mathrm{KTaO}_{3}$ [39].

Figure 3(d) shows the out-of-plane Fermi contour of the metallic state. The absence of dispersion along the $\langle 110\rangle$ direction throughout the complete bulk Brillouin zone demonstrates the near-surface confinement of the conduction band, hence forming a 2DES. The ARPES intensity modulations as a function of out-of-plane momentum, also observed in many other 2DESs $[33,35,36,38,48]$, are a consequence of dipole-transition selection rules from the confined electronic states at the surface of the material $[35,38,52]$. The periodicity of this modulation is approximately determined by the width $L=18 \AA$ of the potential well confining the electrons in the $2 \mathrm{DES}$, which yields $2 \pi / L \approx 0.35 \AA$, in good agreement with the value inferred from Fig. 3(d)—see Appendix F for details. Recalling that the in-plane Fermi surface is a circle, the corresponding Fermi surface in 3D $k$ space would be a cylinder. Our combined findings prove thus the realization of an $s$-derived 2DES on a rutile structure oxide. Following the Luttinger theorem, the corresponding $2 \mathrm{D}$ carrier density $\left(n_{2 D}\right)$ can be extracted from the area $\left(A_{F}\right)$ of the in-plane contour as $n_{2 D}=A_{F} /\left(2 \pi^{2}\right)=(9.4 \pm 0.2) \times 10^{12} \mathrm{~cm}^{-2}$. This is about five times smaller than the carrier density found in analogous 2DESs created by oxygen vacancies at the surface of other binary oxides, such as $\mathrm{TiO}_{2}(001)$-anatase [35] or $\mathrm{ZnO}(000 \overline{1})$ [33], and up to 20 times smaller than the density of the 2DES at the $\mathrm{SrTiO}_{3}(001)$ surface $[36,37,42]$.

\section{B. Tunability of the 2DES \\ 1. Temperature dependence}

We now show two simple methods for tuning the carrier density of the 2DES in $\mathrm{SnO}_{2}$ : controlled temperature variations and surface deposition of europium $(\mathrm{Eu})$ or aluminium (Al). Figures 4(a)-4(g) show the energy-momentum dispersion of the 2DES as the temperature gradually increases from $20 \mathrm{~K}$ to $300 \mathrm{~K}$. The succession of images reveals that the metallic state continuously shifts upwards in energy upon increasing $T$. The corresponding decrease of the Fermi momenta translates into a reduction of the $2 \mathrm{D}$ carrier density. A quantitative analysis of the $k_{F}$ values obtained from the MDCs, presented in Table I, shows a 50\% reduction of the carrier density at $150 \mathrm{~K}$ with respect to $20 \mathrm{~K}$. We highlight the clear 2DES fingerprint at room temperature, but we note that there is an uncertainty whether the electronic band lies above or below $E_{F}$ at such high temperatures because the band bottom is masked by the thermal broadening. Temperaturedependent transport measurements may shed light on this issue. Nevertheless, the robustness of the 2DES at high temperatures is of obvious importance for technological applications. We underline that the observed tunability in carrier density by means of temperature variations is well controlled and, to a great extent, reversible.

After heating the sample from $16 \mathrm{~K}$ to $300 \mathrm{~K}$ in order to decrease the 2D carrier density as shown in Fig. 4, we decreased the temperature back to $15 \mathrm{~K}$ in order to check the reversibility of the phenomenon. Figure 5 and Table I show indeed that the 2D carrier density increases as the temperature decreases. After a full thermal cycle, the final $k_{F}$ value is 
(a) $20 \mathrm{~K}$

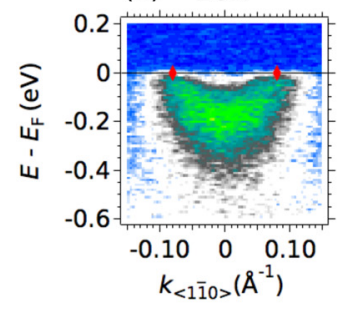

(b) $50 \mathrm{~K}$

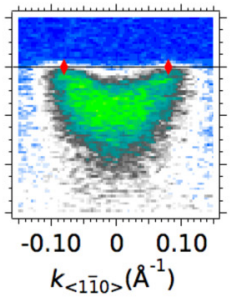

(c) $100 \mathrm{~K}$

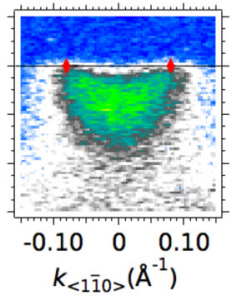

(d) $150 \mathrm{~K}$

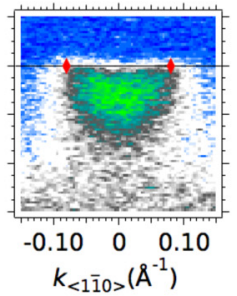

(e) $215 \mathrm{~K}$

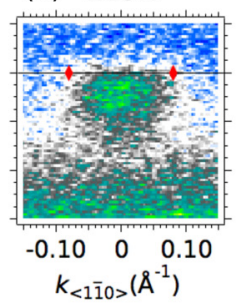

(f) $250 \mathrm{~K}$

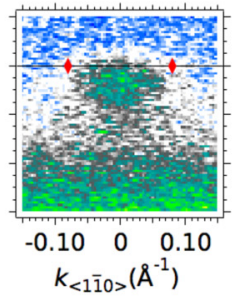

(g) $300 \mathrm{~K}$

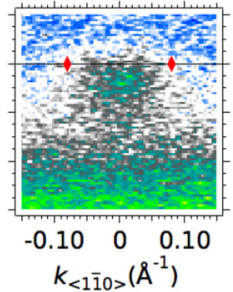

FIG. 4. Energy-momentum dispersion of the $2 \mathrm{DES}$ on $\mathrm{SnO}_{2}(110)$ during a gradual increase of the temperature from $20 \mathrm{~K}$ (a) to $300 \mathrm{~K}$ (g). Red markers indicate the Fermi momenta at $20 \mathrm{~K}$ inferred from the MDC at Fermi level. As the temperature increases, the 2DES state moves up in energy, showing a gradual reduction of the Fermi momenta and the carrier density. Data were acquired with $h v=88 \mathrm{eV}$ and $\mathrm{LH}$ polarization.

close to the original one, although the recovery is incomplete, similar to previous observations for $\mathrm{SrTiO}_{3}$ [53].

The observed temperature dependence of the 2DES carrier density in $\mathrm{SnO}_{2}$ might be due to temperature-induced variations in its dielectric constant [54], which would affect the strength of the confining potential well and/or to thermalinduced migration of oxygen atoms from the bulk to the surface. A thorough exploration of this issue is left open to future works. In this sense, the reason behind the incomplete recovery of the 2DES after a warming-cooling cycle might be the diffusion, while the sample is heated, of oxygen atoms from the bulk to the surface that irreversibly replenish some of the oxygen vacancies.

\section{Surface deposition of Eu or Al}

The second method for modifying the 2D carrier density, namely surface deposition of $\mathrm{Eu}$ or $\mathrm{Al}$, is inspired by our recent studies showing that some metallic adatoms on the surface of transition metal oxides act as efficient reduction agents, pumping out the near-surface oxygen atoms, thus producing $2 \mathrm{DES}$ capped by a layer of the adatom native oxide $[36,40]$. Figure 6 summarizes all the pertinent spectral changes after the deposition of $1 \mathrm{ML}$ of elemental $\mathrm{Eu}$ or $2 \AA$ of elemental Al. Besides the clear oxidation of $\mathrm{Al}$ as indicated in Fig. 6(a), or the formation of $\mathrm{Eu}^{+2}$ as indicated in Fig. 6(c), one also observes, as shown in Fig. 6(b), that the bundle of $\mathrm{SnO}_{2}-4 d$ core levels is drastically modified, developing two new peaks at its low binding energy side. The energy of these new peaks matches well with the $4 d$ spin-orbit-split doublet of metallic Sn, while their seemingly "inverted" branching ratio indeed agrees well with previous experiments on partially oxidized tin [55], all of which indicates that near-surface $\mathrm{Sn}$ atoms have been deprived of some of their oxygen neighbors. A detailed quantitate analysis of these peaks by numerical fitting is given in Appendix C. Note that in Fig. 6(c), the additional peak above the valence band after Eu deposition, which we assign to the $\mathrm{Eu}^{+2} 4 f$ core level, did not appear after $\mathrm{Al}$ deposition as expected.

Combining all these results, we can conclude that: (1) the new pair of peaks after $\mathrm{Eu}$ or $\mathrm{Al}$ deposition are not related to Eu core levels, but rather to the doublet of metallic $\mathrm{Sn} 4 d$ core levels; (2) the additional peak above the valence band after Eu deposition is not an in-gap state due to oxygen vacancies, but rather the $\mathrm{Eu}^{+2} 4 f$ core level.

The presence of Sn atoms with a lower oxidation number than in the pure surface is an indication that $\mathrm{Al}$ or Eu have been effective in removing the near-surface oxygen atoms by a redox reaction, hence driving the emergence of a $2 \mathrm{DES}$, as shown, respectively, in Figs. 6(d) and 6(e), similar to the $\mathrm{AlO}_{x} / \mathrm{SrTiO}_{3}$ and $\mathrm{EuO} / \mathrm{SrTiO}_{3}$ systems $[36,40]$.

As shown in Fig. 6(f), the Fermi momenta of the 2DES at the $\mathrm{AlO}_{x} / \mathrm{SnO}_{2}$ interface (green) or the $\mathrm{EuO} / \mathrm{SnO}_{2}$ interface (blue) are larger than the one at the bare $\mathrm{SnO}_{2}$ surface (red). The corresponding $2 \mathrm{D}$ carrier density in $\mathrm{EuO} / \mathrm{SnO}_{2}$, deduced from the observed Fermi momenta $k_{F}=(0.116 \pm$ $0.001) \AA^{-1}$, is now $(2.15 \pm 0.04) \times 10^{13} \mathrm{~cm}^{-2}$, implying a twofold increase with respect to the bare surface of $\mathrm{SnO}_{2}$, while in $\mathrm{AlO}_{x} / \mathrm{SnO}_{2}$, the $2 \mathrm{D}$ carrier density increases around $40 \%$ to $(1.32 \pm 0.03) \times 10^{13} \mathrm{~cm}^{-2}$. This enhancement of $n_{2 D}$ beyond the saturation limit presented by the bare surface under UV irradiation has not been observed in other 2DES created by redox reactions at metal-oxide interfaces $[36,40]$ and implies that the carrier density of the 2DES in $\mathrm{SnO}_{2}$ can be tuned via adatom deposition. As shown in the Appendix D, the $2 \mathrm{D}$ character of the metallic state at the $\mathrm{EuO} / \mathrm{SnO}_{2}$ interface is confirmed by the absence of out-of-plane dispersion.

One step further, 2 ML elementary Eu can be deposited at the surface of $\mathrm{SnO}_{2}$ (Appendix E). As a final result, a higher

TABLE I. Fermi wave vectors of the 2DES in $\mathrm{SnO}_{2}(110)$ at different temperatures. The corresponding carrier densities are calculated using the Luttinger theorem. A star denotes that the corresponding temperature has been reached starting from a higher value (i.e., during a cooling process). The temperature uncertainty is $\pm 2 \mathrm{~K}$.

\begin{tabular}{lccccc}
\hline \hline$T(\mathrm{~K})$ & 20 & 50 & 100 & 150 & $100^{*}$ \\
\hline$k_{F}\left(10^{-3} \AA^{-1}\right)$ & $85 \pm 1$ & $80 \pm 1$ & $71 \pm 1$ & $60 \pm 2$ & $51 \pm 2$ \\
$n_{2 D}\left(10^{12} \mathrm{~cm}^{-2}\right)$ & $11.5 \pm 0.3$ & $10.2 \pm 0.3$ & $8.0 \pm 0.2$ & $5.7 \pm 0.4$ & $4.1 \pm 0.3$ \\
\hline \hline
\end{tabular}




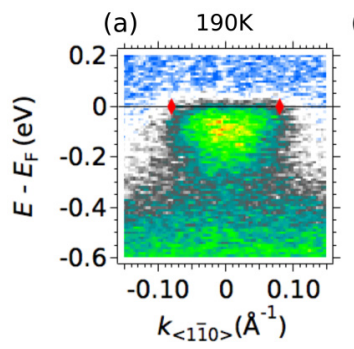

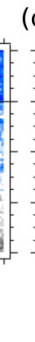

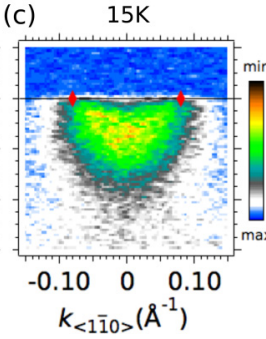

FIG. 5. Energy-momentum dispersion of the 2DES at different temperatures decreasing from $193 \mathrm{~K}$ (a) to $15 \mathrm{~K}$ (c). As in Fig. 4, red markers denote the Fermi wave vectors of the 2DES at $20 \mathrm{~K}$, before the beginning of annealing. The temperature uncertainty is $\pm 2 \mathrm{~K}$.

doublet of the metallic Sn $4 d$ peaks is observed, while no additional metallic states possibly from metallic Eu are detected, suggesting that the deposited $2 \mathrm{ML}$ Eu are fully oxidized. However, the so-formed 2DES shows the same carrier density obtained with the 1 ML Eu capping, a saturation phenomenon also observed in the $\mathrm{EuO} / \mathrm{SrTiO}_{3}$ system [40]. Additionally, as $2 \mathrm{ML}$ of EuO are ferromagnetic [40], one could expect to induce a magnetization of the underlying 2DES, which can be interesting for applications in spintronics.

\section{DISCUSSION}

We now turn to the origin of the 2DES on $\mathrm{SnO}_{2}$. The widely-accepted mechanism of formation of 2DESs at the surface of transition metal oxides, such as $\mathrm{SrTiO}_{3}, \mathrm{TiO}_{2}$, $\mathrm{KTaO}_{3}, \mathrm{CaTiO}_{3}$, and $\mathrm{ZnO}$, is the creation of oxygen vacancies in the near-surface region, induced either by UV/X incoming photons, or by a redox reaction between the oxide substrate and a reducing metallic agent evaporated at its surface $[33,35-38,40,42-48,56]$. Nevertheless, in some oxides, other microscopic phenomena, such as intrinsic electron accumulation and doping with hydrogen impurities may also have an important role for the onset of conductivity [57]. In fact, recent numerical calculations for $\mathrm{SnO}_{2}$ have suggested that even though the (110) surface, which is characterized by an ordered arrangement of $\mathrm{Sn}_{3} \mathrm{O}_{3}$ clusters, contains a deficiency of oxygen atoms, it remains insulating with a small band gap open [3]. Our work shows that the deposition of a reducing metal agent changes dramatically the chemical environment (a)
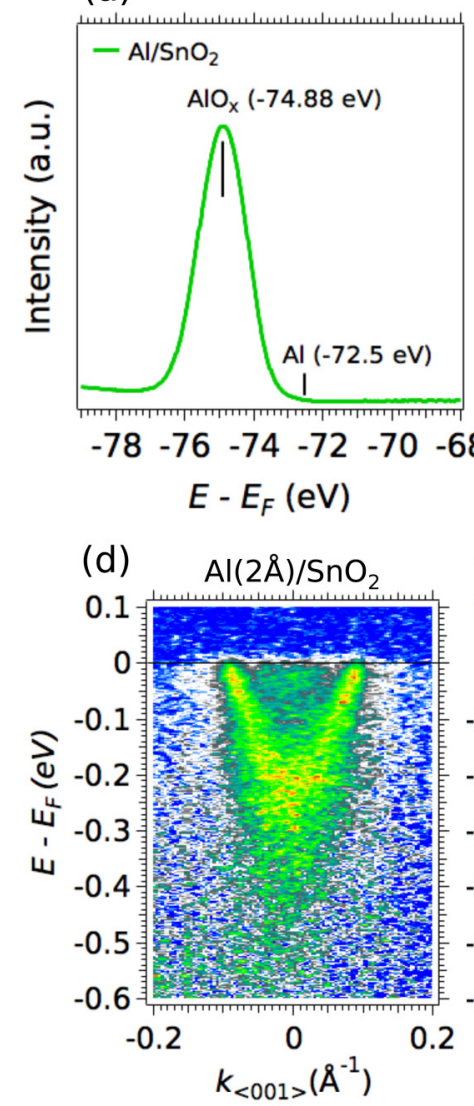

(b)

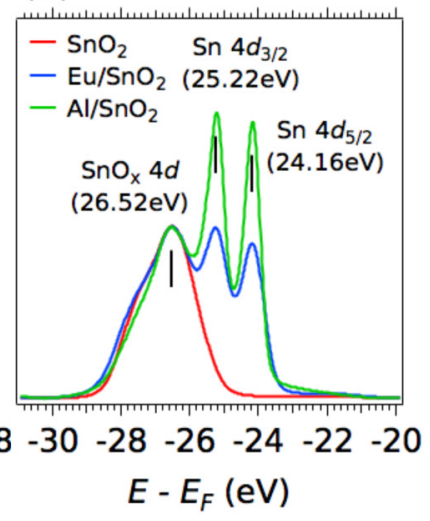

(e) $\mathrm{Eu}(1 \mathrm{ML}) / \mathrm{SnO}_{2}$

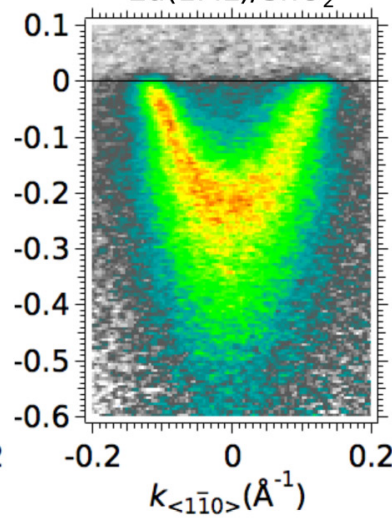

(c)

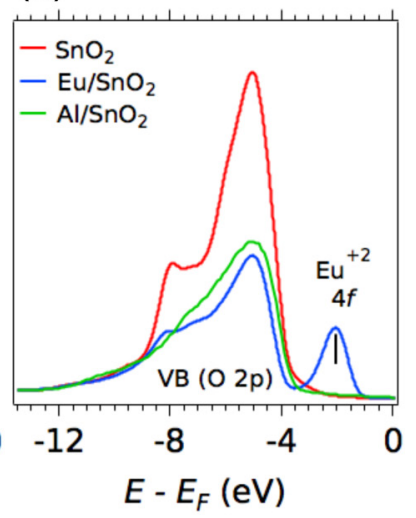

(f)

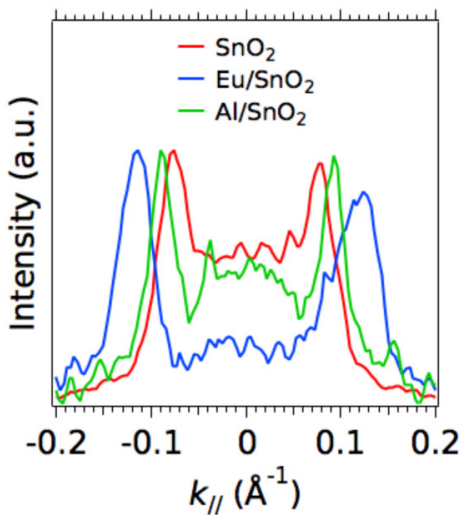

FIG. 6. (a) Al $2 p$ core-level peaks, (b) Sn $4 d$ core-level peaks, and (c) Sn valence band before (red) and after the deposition of $1 \mathrm{ML}$ Eu (blue) or $2 \AA \mathrm{Al}$ (green) on $\mathrm{SnO}_{2}(110)$. (d), (e) Energy-momentum dispersion of the $2 \mathrm{DES}$ measured at the $\mathrm{AlO}_{x} / \mathrm{SnO}_{2}(110)$ interface obtained after deposition of $2 \AA$ of elemental $\mathrm{Al}$, and at the $\mathrm{EuO} / \mathrm{SnO}_{2}(110)$ interface obtained after deposition of $1 \mathrm{ML}$ of elemental Eu, respectively. (f) Comparison of the momentum distribution curves (MDCs) at $E_{F}$ (integrated over $\pm 10 \mathrm{meV}$ ) of the 2DESs at the bare (red), Eu-capped (blue), and Al-capped (green) $\mathrm{SnO}_{2}$ surfaces. All data in this figure were measured at $16 \mathrm{~K}$ with $\mathrm{LH}$ photons. The photon energy was 110 eV for panels (a),(b) and $88 \mathrm{eV}$ for panels (c)-(f). 

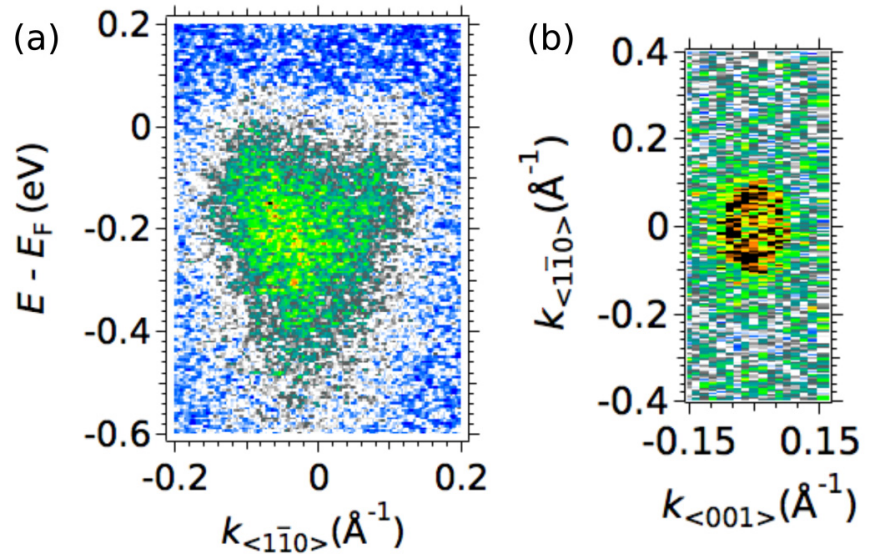

FIG. 7. (a) Experimental energy-momentum dispersion of the 2DES measured at the surface of an as-received $\mathrm{SnO}_{2}$ crystal, i.e., never prepared or cleaned in $U H V$. (b) Corresponding Fermi surface contour around the center of the projected surface Brillouin zone. All data in this figure were acquired at $20 \mathrm{~K}$ using $\mathrm{LH}$ photons of energy $120 \mathrm{eV}$.

of $\mathrm{Sn}$ atoms as some of the latter pass into a lower oxidation state [Fig. 6(a)]. Therefore, the structural model based on $\mathrm{Sn}$ interstitials cannot account for the surface 2DES. On the other hand, a structural model based on the presence of surface oxygen vacancies as suggested in Refs. [10,58] might explain the absence of bonding counterparts to the $\mathrm{Sn}$ atoms and the enhancement of the 2DES.

The 2DES observed at the (110) surface of $\mathrm{SnO}_{2}$ is, to our knowledge, the first of its kind on a rutile structure. In fact, a previous comparison of the near- $E_{F}$ electronic structure of $\mathrm{TiO}_{2}$-rutile and $\mathrm{TiO}_{2}$-anatase has led to the conclusion that surface oxygen vacancies on a rutile structure create excess electrons that remain localized at Ti sites rather than forming a 2DES [35,59]. The present work shows that such a conclusion cannot be generalized to all rutile lattices, and other factors, such as the orbital character of the confined states $\left(d\right.$ for $\mathrm{TiO}_{2}$ vs $s$ for $\mathrm{SnO}_{2}$ ), have to be taken into account to understand the emergence of a 2DES.

Finally, we underline that besides its robustness against different surface reconstructions and high temperatures, the 2DES at the surface of $\mathrm{SnO}_{2}$ even survives exposure to ambient pressure. In fact, being an excellent material for heterogeneous catalysis, the surface of $\mathrm{SnO}_{2}$ is expected to easily adsorb and desorb gas molecules. Thus, in order to test its catalytic properties, we studied the electronic structure of the $\mathrm{SnO}_{2}$ (110) surface without prior preparation or cleaning in $U H V$, and using the UV beam at beamline $2 \mathrm{~A}$ of KEK$\mathrm{PF}$, whose low brilliance $\left(5 \times 10^{7}\right.$ photons $\left.\mathrm{s}^{-1} \mu \mathrm{m}^{-2}\right)$, about 100 times smaller than other photoemission beamlines in third generation synchrotrons, has been previously shown to strongly reduce or even inhibit the photoinduced creation of oxygen vacancies in other oxides [60]. As demonstrated in Fig. 7, the characteristic parabolic dispersion and circular Fermi surface of the 2DES in $\mathrm{SnO}_{2}$ can be observed even under these adverse measurement conditions-note that even the slightest exposure to moderate vacuum would normally make the surface unsuitable for ARPES measurements, due to the strong surface sensitivity of the technique. We note furthermore that the 2DES was observed immediately after illumination with the low-brilliance UV beam, and no evolution of its carrier density was evident even after several hours of measurements.

The Fermi momentum of such a 2DES intrinsically present at the $\mathrm{SnO}_{2}$ surface, determined from the MDC peaks at $E_{F}$, is $k_{F}=(0.077 \pm 0.003) \AA^{-1}$, which is comparable to the Fermi momentum of the 2DES observed at the UHV-prepared surface shown in Fig. 3. These results show that, either under the normal conditions of ambient pressure, temperature and illumination, or the exposure to low-brilliance UV light, a $2 \mathrm{DES}$ in $\mathrm{SnO}_{2}$ can be readily observed despite the absence of surface treatment, suggesting that the catalytic properties of this material protect, or might even induce, such a 2DES, and making of $\mathrm{SnO}_{2}$ a unique candidate for future technological applications.

\section{CONCLUSIONS}

In conclusion, we observed a metallic 2DES at the (110) surface of $\mathrm{SnO}_{2}$ by means of ARPES. Its carrier density can be enhanced by the deposition of elemental $\mathrm{Al}$ or $\mathrm{Eu}$, and reduced by a controlled temperature increase, while the 2DES remains robust against different surface reconstructions, high temperatures and exposure to ambient pressure. The spectral fingerprints of the 2DES show an appreciable electron-phonon interaction, with the system always lying in the Fermi liquid regime (Appendix $\mathrm{G}$ ) due to the small dielectric constant of $\mathrm{SnO}_{2}$. The strong effect of a reducing metal agent such as $\mathrm{Al}$ or $\mathrm{Eu}$ on the emergence and enhancement of the 2DES proves that surface oxygen vacancies are at the origin of the 2DES in $\mathrm{SnO}_{2}$. Our results provide important insights on the long-withstanding debate on the origin of the $n$-type conductivity of $\mathrm{SnO}_{2}$ and prove that both the crystal structure and the orbital origin of the conduction electrons are decisive for the emergence of a $2 \mathrm{DES}$ in oxides.

\section{ACKNOWLEDGMENTS}

Work at CSNSM and ISMO was supported by public grants from the Centre National de la Recherche Scientifique (CNRS, project PICS FermiAds No. 272651), the French National Research Agency (ANR, project LACUNES No. ANR-13-BS04-0006-01), and the "Laboratoire d'Excellence Physique Atomes Lumière Matière" (LabEx PALM, projects ELECTROX, 2DEGS2USE and 2DTROX) overseen by the ANR as part of the "Investissements d'Avenir" program (reference: ANR-10-LABX-0039). Work at KEK-PF was supported by Grants-in-Aid for Scientific Research (Nos. $16 \mathrm{H} 02115$ and 16KK0107) from the Japan Society for the Promotion of Science (JSPS). Experiments at KEK-PF were performed under the approval of the Program Advisory Committee (Proposals 2016G621, 2015S2005, and 2018S2004) at the Institute of Materials Structure Science at KEK.

\section{APPENDIX A: ARPES RESULTS WITH LINEAR VERTICAL POLARIZATION}

The majority of the data shown in the main text were acquired with linear horizontal (LH) light polarization. For 

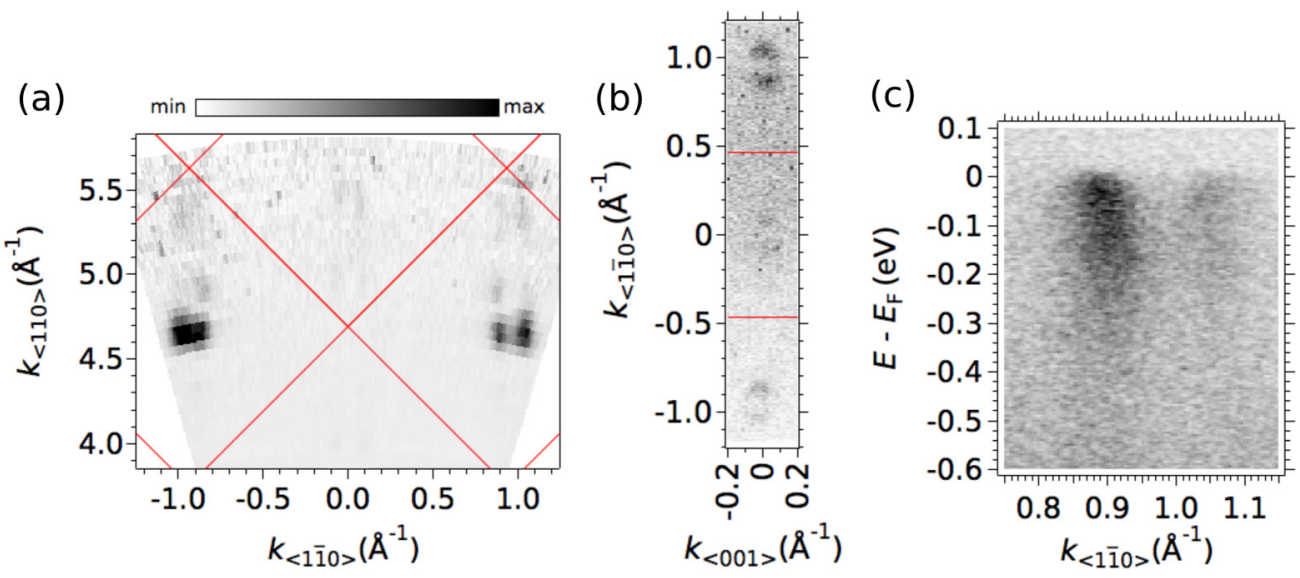

FIG. 8. (a) Out-of-plane Fermi surface map of $\mathrm{SnO}_{2}$ (110) measured by changing the photon energy between 50 and 120 eV in steps of $1 \mathrm{eV}$. (b) In-plane Fermi surface of $\mathrm{SnO}_{2}(110)$ measured with $h v=88 \mathrm{eV}$. Red lines mark the borders of the projected surface Brillouin zone. (c) Energy-momentum dispersion of the 2DES measured with $h v=88 \mathrm{eV}$, corresponding to an out-of-plane momentum in the upper Brillouin zone of (b). All data in this figure were acquired at $16 \mathrm{~K}$ using linear vertical polarization. An inner potential of $10 \mathrm{eV}$ was used in the calculation of the out-of-plane momentum.

the sake of completion we present here the main results obtained on the bare (110) surface of $\mathrm{SnO}_{2}$ using linear vertical (LV) polarization. Figure 8 shows the out-of-plane Fermi surface contours, the in-plane Fermi surface contours, and the energy dispersion of the metallic 2DES as probed by LV photons in panels (a), (b), and (c), respectively. A comparison of Figs. 8(a) and 3(d) of the main text reveals a clear polarization selection at normal emission (i.e., $k_{\|}=0$ ) where there is almost no photoemission intensity with LV photons. Moreover, there is a strong suppression of spectral weight along the $\langle 001\rangle$ directions. These intensity variations are due to the $s$ orbital character of the 2DES state [51].

\section{APPENDIX B: GIBBS FREE ENERGIES OF RELEVANT REDOX REACTIONS}

We now consider the Gibbs free energies for the redox reactions relevant to our experiments, namely the ones needed to form $\mathrm{SnO}_{2}, \mathrm{Al}_{2} \mathrm{O}_{3}$, and $\mathrm{EuO}$ at $25{ }^{\circ} \mathrm{C}$. According to the database found in the chemistry-reference website [61], we have:

$$
\begin{aligned}
\mathrm{Sn}+\mathrm{O}_{2} & =\mathrm{SnO}_{2}+519.65 \mathrm{~kJ} / \mathrm{mol}, \\
\mathrm{Eu}+(1 / 2) \mathrm{O}_{2} & =\mathrm{EuO}+556.89 \mathrm{~kJ} / \mathrm{mol}, \\
2 \mathrm{Al}+(3 / 2) \mathrm{O}_{2} & =\mathrm{Al}_{2} \mathrm{O}_{3}+1581.97 \mathrm{~kJ} / \mathrm{mol},
\end{aligned}
$$

Thus,

$$
\begin{aligned}
\mathrm{SnO}_{2}+2 \mathrm{Eu} & =\mathrm{Sn}+2 \mathrm{EuO}+594.13 \mathrm{~kJ} / \mathrm{mol}, \\
3 \mathrm{SnO}_{2}+4 \mathrm{Al} & =3 \mathrm{Sn}+2 \mathrm{Al}_{2} \mathrm{O}_{3}+1604.99 \mathrm{~kJ} / \mathrm{mol},
\end{aligned}
$$

So, instead of a simple picture of Eu or Al ionization, these exothermic redox reactions will happen spontaneously once $\mathrm{Eu}$ or $\mathrm{Al}$ is deposited at the $\mathrm{SnO}_{2}$ surface. This conclusion is consistent with our XPS data and analysis.

\section{APPENDIX C: QUANTITATE ANALYSIS OF SN $4 d$ CORE LEVEL PEAKS BY NUMERICAL FITTING}

We further analyzed the $\mathrm{Sn} 4 d$ core levels measured after $\mathrm{Al}(2 \AA)$ or $\mathrm{Eu}(1 \mathrm{ML})$ deposition at the $\mathrm{SnO}_{2}(110)$ surface by numerical fitting with Lorentzian peaks and a Shirley background as shown in Fig. 9. As demonstrated by previous experiments [55], exposure of a clean tin surface to oxygen results in the appearance of an oxidized component at the high-binding-energy side of the metallic Sn $4 d$ doublet, composed of a bundle of four peaks: the $\mathrm{Sn}^{2+}$ and $\mathrm{Sn}^{4+}$ doublets. Thus, to fit our data we use three doublets $\left(\mathrm{Sn}^{0}, \mathrm{Sn}^{2+}\right.$, and $\mathrm{Sn}^{4+}$ states), constraining their branching ratio to $0.65 \pm 0.5$, within $10 \%$ of the value of $2 / 3$ expected for a $d$ doublet, their energy difference to $1.1 \pm 0.05 \mathrm{eV}$, corresponding to the $4 d$ splitting in metallic tin [55], and keeping the same
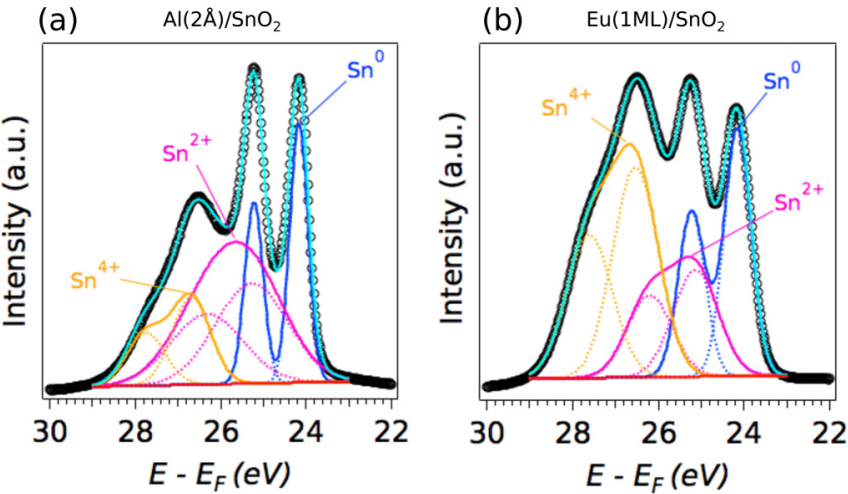

FIG. 9. Numerical fitting of the $\mathrm{SnO}_{2} 4 d$ core levels after deposition of (a) $2 \AA$ of $\mathrm{Al}$, and (b) $1 \mathrm{ML}$ of Eu at the $\mathrm{SnO}_{2}(110)$ surface. Black open circles: XPS data. Dashed and full orange lines: Lorentzian peaks and doublet for the $\mathrm{Sn}^{4+}$ component. Dashed and full purple lines: Lorentzian peaks and doublet for the $\mathrm{Sn}^{2+}$ component. Dashed and full dark blue lines: Lorentzian peaks and doublet for the $\mathrm{Sn}^{0}$ component. Red: Shirley background. Sky blue line: Overall fit. 


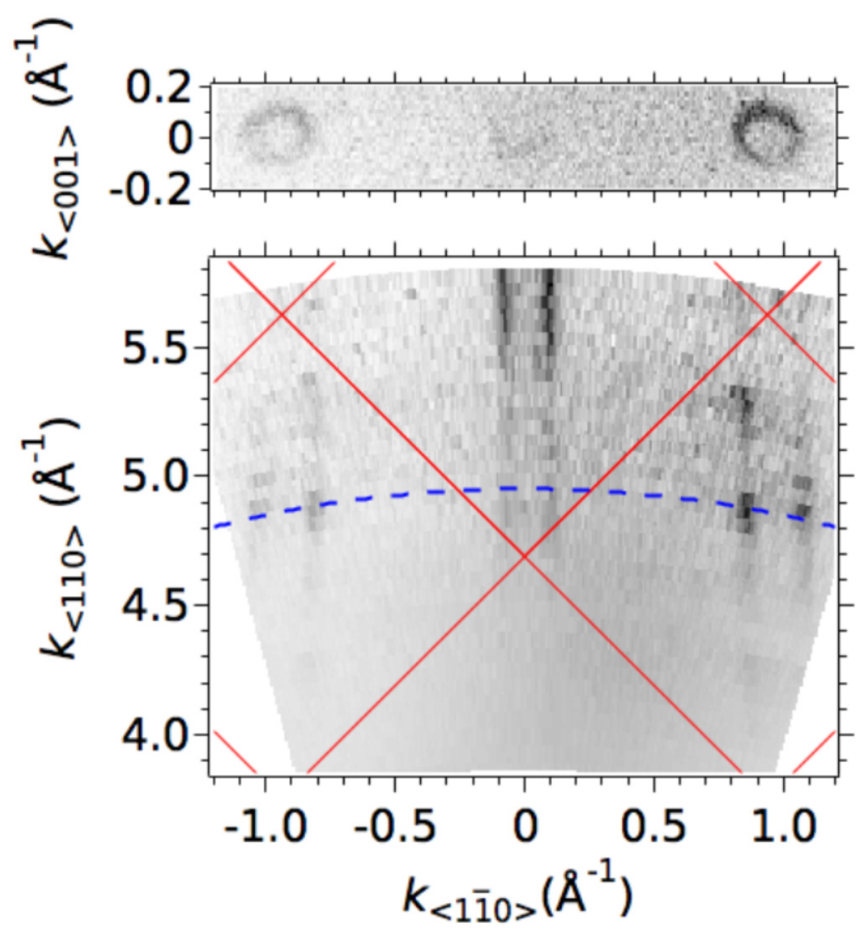

FIG. 10. Top panel: in-plane Fermi surface of $\operatorname{Eu}(1 \mathrm{ML}) /$ $\mathrm{SnO}_{2}(110)$ measured with $h v=88 \mathrm{eV}$. Bottom panel: out-of-plane Fermi surface map of $\mathrm{Eu}(1 \mathrm{ML}) / \mathrm{SnO}_{2}(110)$ measured by a stepwise change of $1 \mathrm{eV}$ of the photon energy between 50 and $120 \mathrm{eV}$. The blue dashed arc shows the cut in reciprocal space corresponding to $h v=88 \mathrm{eV}$, using an inner potential $V_{0}=10 \mathrm{eV}$.

broadening for the peaks of each doublet. As seen in Fig. 9, the overall fit describes very well the data, confirming the effective reduction, and concomitant formation of oxygen vacancies, in the $\mathrm{SnO}_{2}$ surface after $\mathrm{Al}$ or Eu deposition.

\section{APPENDIX D: FERMI SURFACES OF THE 2DES AT THE INTERFACE EUO(1 ML)/SnO}

Figure 10 shows the in-plane and out-of-plane Fermi surfaces obtained at the $\mathrm{EuO}(1 \mathrm{ML}) / \mathrm{SnO}_{2}$ interface after deposition of $1 \mathrm{ML}$ of metallic Eu on the $\mathrm{SnO}_{2}(110)$ surface. The in-plane periodicity of the Fermi circles corresponds to that of the projected bulk Brillouin zone, while the absence of out-of-plane dispersion demonstrates the $2 \mathrm{D}$ character of the confined state.

\section{APPENDIX E: 2 ML ELEMENTAL EU CAPPING AT THE $\mathrm{SnO}_{2}(110)$ SURFACE}

According to a previous work in the $\mathrm{EuO} / \mathrm{SrTiO}_{3}$ system [40], $\mathrm{EuO}(1 \mathrm{ML}) / \mathrm{SrTiO}_{3}$ shows a paramagnetic behavior while $\mathrm{EuO}(2 \mathrm{ML}) / \mathrm{SrTiO}_{3}$ shows a ferromagnetic behavior, even though the carrier density remains the same in both cases, indicating a saturation in the creation of itinerant electrons from oxygen vacancies at the surface. Inspired by this work, we deposited $2 \mathrm{ML}$ pure $\mathrm{Eu}$ on $\mathrm{SnO}_{2}$ to check whether this Eu coverage will also be fully oxidized and explore potential changes in the 2DES. (a)

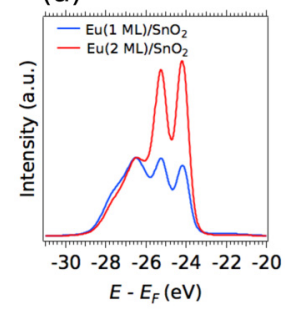

(b)

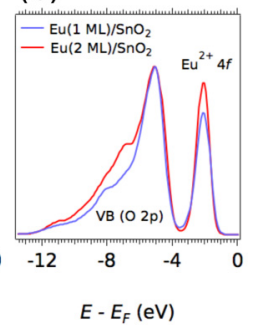

(c)

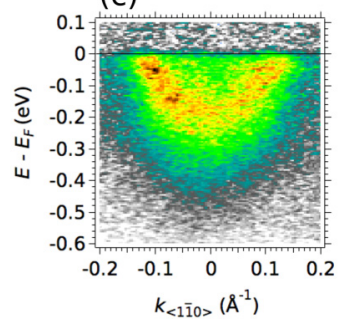

FIG. 11. (a),(b) Sn $4 d$ core-level peaks and valence band, respectively, after the deposition of $1 \mathrm{ML}$ (blue) and $2 \mathrm{ML}$ (red) Eu on $\mathrm{SnO}_{2}(110)$. The photon energy used was $110 \mathrm{eV}$. (c) Energymomentum dispersion of the 2DES at the $\mathrm{Eu} / \mathrm{SnO}_{2}(110)$ interface obtained after deposition of 2 ML of elemental Eu measured with LH photons at $h v=88 \mathrm{eV}$.

The observation of a larger metallic Sn $4 d$ doublet in Fig. 11(a) and a larger $\mathrm{Eu}^{+2} 4 f$ peak in Fig. 11(b) suggest that more oxygen vacancies were created after deposition of $2 \mathrm{ML}$ $\mathrm{Eu}$ on the $\mathrm{SnO}_{2}$ surface, compared to $1 \mathrm{ML}$ Eu deposition. In both cases, the XPS profile lines show the same shape without additional peaks appearing, indicating that Eu is still mainly oxidized into $\mathrm{Eu}^{+2}$. The 2DES resulting after deposition of $2 \mathrm{ML} \mathrm{Eu}$ on the $\mathrm{SnO}_{2}(110)$ surface, presented in Fig. 11(c), shows the same carrier density and effective mass as the 2DES at the interface of $\mathrm{Eu}(1 \mathrm{ML}) / \mathrm{SnO}_{2}$. An analogous saturation was reported in the $\mathrm{EuO} / \mathrm{SrTiO}_{3}$ system [40]. These result also indicate that the $2 \mathrm{ML} \mathrm{Eu}$ are fully oxidized.

\section{APPENDIX F: EXTRACTING THE 2DES PARAMETERS}

We now present a further analysis of the 2DES dispersion after the deposition of Eu in order to extract the parameters of the confinement surface potential. Figure 12 shows the

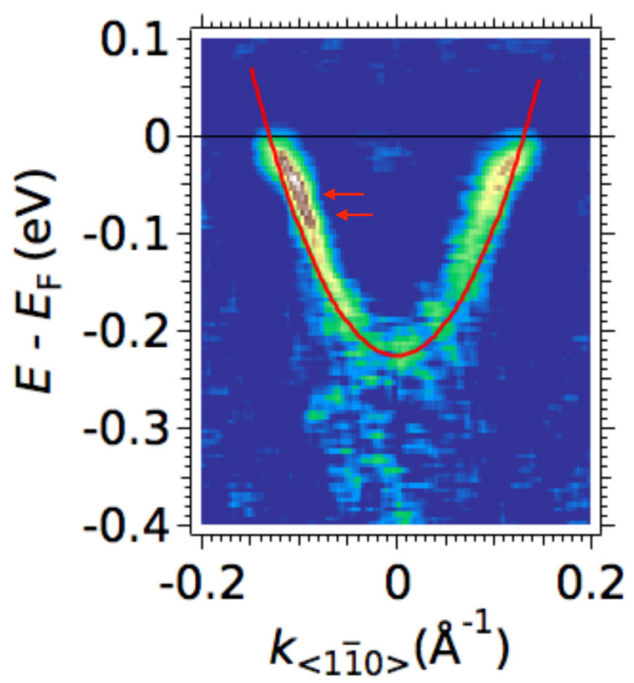

FIG. 12. Curvature of the experimental energy-momentum dispersion shown in Fig. 3(c) of the main text together with a parabolic fit of the form $E=\left(\hbar^{2} / 2 m^{\star}\right) k^{2}+E_{b}$, with $E_{b}=-225 \mathrm{meV}$ and $m^{\star}=0.29 m_{e}$. Two kinks at $59 \mathrm{meV}$ and $79 \mathrm{meV}$ are the fingerprints of electron-phonon interaction. 
2D curvature [62] of the spectral intensity previously shown in Fig. 6(e) of the main text. A parabolic fit of the energy dispersion, in the form $E=\left(\hbar^{2} / 2 m^{\star}\right) k^{2}+E_{b}$, yields a band bottom $E_{b}=-225 \mathrm{meV}$ and an effective mass $m^{\star}=0.29 m_{e}$ [63]. We assume that the first excited state $(n=1)$ of the confining quantum well, not directly observed in our data, lies just above $E_{F}$. This will provide an upper bound for the width of the quantum well. Taking, for simplicity, a wedge-shaped potential well, the values (in $\mathrm{eV}$ ) of the discrete energy levels $E_{n}$ are given by [37,41]:

$$
E_{n}=V_{0}+9 \times 10^{-7}\left(\frac{m_{e}}{m^{\star}}\right)^{1 / 3}\left(n+\frac{3}{4}\right)^{2 / 3} F^{2 / 3},
$$

with $V_{0}$ the energy depth of the quantum well $(\mathrm{eV}), m^{\star}$ the effective mass of the confined electrons, and $F$ the strength of the electric field generating the confining potential $(\mathrm{V} / \mathrm{m})$.

With the $n=0$ subband (ground state) at $225 \mathrm{meV}$ below $E_{F}$, and the $n=1$ subband (first excited state) at the Fermi level, the electric field strength can be calculated to be $F=$ $122 \mathrm{MV} / \mathrm{m}$. This yields $V_{0} \approx 500 \mathrm{meV}, L \approx 18 \AA$ (i.e., three unit cells) for the spatial extension of the wave function in the ground state of the 2DES, the main part of which thus lies 1-2 unit cells below the surface, and a maximum width of the confining potential $L_{\max }=42 \AA$ (six unit cells). These parameters are similar to the characteristics of the textbook 2DES observed at the (001) surface of $\mathrm{SrTiO}_{3}[37,41,42]$.

\section{APPENDIX G: ELECTRON-PHONON INTERACTION}

The fingerprint of the electron-phonon interaction is a kink in the experimental dispersion that corresponds to the characteristic energy of the phonon. After the deposition of Eu, two such kinks are observed in the energy dispersion of the 2DES at the (110) surface of $\mathrm{SnO}_{2}$ (Fig. 12). The corresponding phonon energies are $59 \mathrm{meV}\left(E_{g}\right.$ mode $)$ and $79 \mathrm{meV}\left(A_{1 g}\right.$ mode) [64-66]. In the presence of electron-phonon coupling, the predominant interactions vary as a function of the carrier density, giving rise to two different regimes: the polaronic regime at low carrier densities, where the long-range Fröhlich interaction prevails, and the Fermi liquid regime at high carrier densities, where the long range interaction is suppressed by strong electronic screening [49,50].

The critical carrier density between these two regimes can be estimated by balancing the relevant phonon energy and the surface plasma frequency $\omega_{s}$ given by the carrier density $n$ [67]:

$$
\omega_{s}=\sqrt{\frac{n e^{2}}{\varepsilon_{0}\left(\varepsilon_{\infty}+1\right) m^{\star}}},
$$

where $\varepsilon_{0}$ is the vacuum permittivity, $\varepsilon_{\infty}$ is the dielectric constant in high frequency limit, and $m^{\star}$ is the carriers' effective mass. Given that $\varepsilon_{\infty}=5$ and $m^{\star}=(0.24 \pm 0.02) m_{e}$ for $\mathrm{SnO}_{2}$, we can use a relevant phonon energy cutoff of $80 \mathrm{meV}$, and the aforementioned spatial extension of the confining potential $\left(L_{\max }=42 \AA\right)$ to estimate a critical carrier density $n_{c} \approx 2.81 \times 10^{12} \mathrm{~cm}^{-2}$. This rather low critical carrier density is mainly due to the small dielectric constant $\varepsilon_{\infty}$ of $\mathrm{SnO}_{2}$ and means that the 2DESs both at the bare $\mathrm{SnO}_{2}$ surface and at the $\mathrm{EuO} / \mathrm{SnO}_{2}$ interface lie in the Fermi liquid regime as their corresponding carrier concentrations are up to one order of magnitude larger than the critical one. This is confirmed in the experimental data by the absence of the spectroscopic fingerprint of Fröhlich polarons: replica bands at higher binding energies [49,50]. This is in stark contrast with the 2DESs on $\mathrm{SrTiO}_{3}$ and $\mathrm{TiO}_{2}$ surfaces where clear band satellites were observed at similar carrier densities owing to the large dielectric constant of the corresponding materials $[49,50,59]$.
[1] M. Batzill and U. Diebold, Phys. Chem. Chem. Phys. 9, 2307 (2007).

[2] M. Batzill, K. Katsiev, and U. Diebold, Surf. Sci. 529, 295 (2003).

[3] L. R. Merte, M. S. Jørgensen, K. Pussi, J. Gustafson, M. Shipilin, A. Schaefer, C. Zhang, J. Rawle, C. Nicklin, G. Thornton et al., Phys. Rev. Lett. 119, 096102 (2017).

[4] Catalysis and Chemical Processes, edited by R. Pearce and W. R. Patterson (Wiley, New York, 1981).

[5] M. Sinner-Hettenbach, Göthelid, T. Weiss, N. Barsan, U. Weimar, H. von Schenck, L. Giovanelli, and G. L. Lay, Surf. Sci. 499, 85 (2002).

[6] S. Semancik and D. F. Cox, Sens. Actu. 12, 101 (1987).

[7] J. Watson, Sens. Actu. 5, 29 (1984).

[8] D. F. Cox, T. B. Fryberger, and S. Semancik, Surf. Sci. 224, 121 (1989).

[9] M. Batzill and U. Diebold, Prog. Surf. Sci. 79, 47 (2005).

[10] F. H. Jones, R. Dixon, J. S. Foord, R. G. Egdell, and J. B. Pethica, Surf. Sci. 376, 367 (1997).

[11] F. J. Berry, Adv. Catal. 30, 97 (1981).
[12] M. Batzill, K. Katsiev, J. M. Burst, U. Diebold A. M. Chaka, and B. Delley, Phys. Rev. B 72, 165414 (2005).

[13] P. G. Harrison, C. Bailey, and W. Azelee, J. Catal. 186, 147 (1999).

[14] F. Solymosi and J. Kiss, J. Catalysis 41, 202 (1976).

[15] M. J. Fuller and M. E. Warwick, J. Catalysis 29, 441 (1973).

[16] M. A. Mäki-Jaskari and T. T. Rantala, Phys. Rev. B 64, 075407 (2001).

[17] R. G. Egdell, J. Rebane, T. J. Walker, and D. S. L. Law, Phys. Rev. B 59, 1792 (1999).

[18] J. Haines and J. M. Léger, Phys. Rev. B 55, 11144 (1997).

[19] K. Reimann and M. Steube, Solid State Commun. 105, 649 (1998).

[20] J. Robertson, J. Phys. C 12, 4767 (1979).

[21] S. Munnix and M. Schmeits, Phys. Rev. B 27, 7624 (1983).

[22] D. F. Cox, T. B. Fryberger, and S. Semancik, Phys. Rev. B 38, 2072 (1988).

[23] R. G. Egdell, S. Eriksen, and W. R. Flavell, Solid State Commun. 60, 835 (1986).

[24] P. A. Cox, R. G. Egdell, C. Harding, W. R. Patterson, and P. J. Tavener, Surf. Sci. 123, 179 (1982). 
[25] J. Themlin, R. Sporken, J. Darville, R. Caudano, J. M. Gilles, and R. Johnson, Phys. Rev. B 42, 11914 (1990).

[26] R. G. Egdell, S. Eriksen, and W. R. Flavell, Solid State Commun. 44, 837 (1982).

[27] R. G. Egdell, S. Eriksen, and W. R. Flavell, Surf. Sci. 192, 265 (1987).

[28] P. C. Hollamby, P. S. Aldridge, G. Moretti, R. G. Egdell, and W. R. Flavell, Surf. Sci. 280, 393 (1993).

[29] J. Oviedo and M. G. Gillan, Surf. Sci. 467, 35 (2000).

[30] J. A. Marley and R. C. Dockerty, Phys. Rev. 140, A304 (1965).

[31] K. H. L. Zhang, R. G. Egdell, F. Offi, S. Iacobucci, L. Petaccia, S. Gorovikov, and P. D. C. King, Phys. Rev. Lett. 110, 056803 (2013).

[32] L. F. J. Piper, L. Colakerol, P. D. C. King, A. Schleife, J. ZúñigaPérez, P.-A. Glans, T. Learmonth, A. Federov, T. D. Veal, F. Fuchs et al., Phys. Rev. B 78, 165127 (2008).

[33] T. C. Rödel, J. Dai, F. Fortuna, E. Frantzeskakis, P. L. Fèvre, F. Bertran, M. Kobayashi, R. Yukawa, T. Mitsuhashi, M. Kitamura, K. Horiba, H. Kumigashira, and A. F. SantanderSyro, Phys. Rev. Mater. 2, 051601(R) (2018).

[34] R. Yukawa, K. Ozawa, S. Yamamoto, H. Iwasawa, K. Shimada, E. F. Schwier, K. Yoshimatsu, H. Kumigashira, H. Namatame, M. Taniguchi et al., Phys. Rev. B 94, 165313 (2016).

[35] T. C. Rödel, F. Fortuna, F. Bertran, M. Gabay, M. J. Rozenberg, A. F. Santander-Syro, and P. Le Fèvre, Phys. Rev. B 92, 041106(R) (2015).

[36] T. C. Rödel, F. Fortuna, S. Sengupta, E. Frantzeskakis, P. L. Fèvre, F. Bertran, B. Mercey, S. Matzen, G. Agnus, T. Maroutian et al., Adv. Mater. 28, 1976 (2016).

[37] A. F. Santander-Syro, O. Copie, T. Kondo, F. Fortuna, S. Pailhès, R. Weht, X. G. Qiu, F. Bertran, A. Nicolau, A. TalebIbrahimi, P. L. Fèvre, G. Herranz, M. Bibes, N. Reyren, Y. Apertet, P. Lecoeur, A. Barthélémy, and M. J. Rozenberg, Nature (London) 469, 189 (2011).

[38] T. C. Rödel, C. Bareille, F. Fortuna, C. Baumier, F. Bertran, P. L. Fèvre, M. Gabay, O. H. Cubelos, M. J. Rozenberg, T. Maroutian, P. Lecoeur, and A. F. Santander-Syro, Phys. Rev. Appl. 1, 051002 (2014).

[39] C. Bareille, F. Fortuna, T. C. Rödel, F. Bertran, M. Gabay, O. H. Cubelos, A. Taleb-Ibrahimi, P. L. Fèvre, M. Bibes, A. Barthélémy, T. Maroutian, P. Lecoeur, M. J. Rozenberg, and A. F. Santander-Syro, Sci. Rep. 4, 3586 (2014).

[40] P. Lömker, T. C. Rödel, T. Gerber, F. Fortuna, E. Frantzeskakis, P. Le Fèvre, F. Bertran, M. Müller, and A. F. Santander-Syro, Phys. Rev. Mater. 1, 062001 (2017).

[41] E. Frantzeskakis, T. C. Rödel, F. Fortuna, and A. F. SantanderSyro, J. Electron Spectr. Rel. Phenom. 219, 16 (2017).

[42] W. Meevasana, P. D. C. King, R. H. He, S.-K. Mo, M. Hashimoto, A. Tamai, P. Songsiriritthigul, F. Baumberger, and Z.-X. Shen, Nat. Mater. 10, 114 (2011).

[43] A. F. Santander-Syro, C. Bareille, F. Fortuna, O. Copie, M. Gabay, F. Bertran, A. Taleb-Ibrahimi, P. Le Fèvre, G. Herranz, N. Reyren, M. Bibes, A. Barthélémy, P. Lecoeur, J. Guevara, and M. J. Rozenberg, Phys. Rev. B 86, 121107 (2012).

[44] P. D. C. King, R. H. He, T. Eknapakul, P. Buaphet, S.-K. Mo, Y. Kaneko, S. Harashima, Y. Hikita, M. S. Bahramy, C. Bell, Z. Hussain, Y. Tokura, Z.-X. Shen, H. Y. Hwang, F. Baumberger, and W. Meevasana, Phys. Rev. Lett. 108, 117602 (2012).
[45] Z. Wang, Z. Zhong, X. Hao, S. Gerhold, B. Stoger, M. Schmid, J. Sanchez-Barriga, A. Varykhalov, C. Franchini, K. Held, and U. Diebold, PNAS 111, 3933 (2014).

[46] S. McKeown Walker, A. de la Torre, F. Y. Bruno, A. Tamai, T. K. Kim, M. Hoesch, M. Shi, M. S. Bahramy, P. D. C. King, and F. Baumberger, Phys. Rev. Lett. 113, 177601 (2014).

[47] S. McKeown Walker, F. Y. Bruno, Z. Wang, A. de la Torre, S. Ricco, A. Tamai, T. K. Kim, M. Hoesch, M. Shi, M. S. Bahramy, P. D. C. King, and F. Baumberger, Adv. Mater. 27 , 3894 (2015).

[48] T. C. Rödel, M. Vivek, F. Fortuna, P. L. Fèvre, F. Bertran, R. Weht, J. Goniakowski, M. Gabay, and A. F. Santander-Syro, Phys. Rev. B 96, 041121(R) (2017).

[49] C. Chen, J. Avila, E. Frantzeskakis, A. Levy, and M. C. Asensio, Nat. Commun. 6, 8585 (2015).

[50] Z. Wang, S. M. Walker, A. Tamai, Y. Wang, Z. Ristic, F. Y. Bruno, A. De La Torre, S. Riccò, N. Plumb, M. Shi et al., Nat. Mater. 15, 835 (2016).

[51] S. Moser, J. Electron Spectr. Rel. Phenom. 214, 29 (2017).

[52] S. Moser, V. Jovic, R. Koch, L. Moreschini, J.-S. Oh, C. Jozwiak, A. Bostwick, and E. Rotenberg, J. Electron Spectrosc. Relat. Phenom. 225, 16 (2018).

[53] T. C. Rödel, private communication.

[54] A. S. Lanje, S. J. Sharma, R. B. Pode, and R. S. Ningthoujam, Arch. Apll. Sci. Res. 2, 127 (2010).

[55] P. D. Padova, M. Fanfoni, R. Larciprete, M. Mangiantini, S. Priori, and P. Perfetti, Surf. Sci. 313, 379 (1994).

[56] Y. Aiura, I. Hase, H. Bando, T. Yasue, T. Saitoh, and D. S. Desau, Surf. Sci. 515, 61 (2002).

[57] P. King and T. D. Veal, J. Phys. Condens. Matter 23, 334214 (2011).

[58] J. Oviedo and M. J. Gillan, Surf. Sci. 513, 26 (2002).

[59] S. Moser, L. Moreschini, J. Jaćimović, O. S. Barišić, H. Berger, A. Magrez, Y. J. Chang, K. S. Kim, A. Bostwick, E. Rotenberg et al., Phys. Rev. Lett. 110, 196403 (2013).

[60] S. Backes, T. C. Rödel, F. Fortuna, E. Frantzeskakis, P. Le Fèvre, F. Bertran, M. Kobayashi, R. Yukawa, T. Mitsuhashi, M. Kitamura, K. Horiba, H. Kumigashira, R. Saint-Martin, A. Fouchet, B. Berini, Y. Dumont, A. J. Kim, F. Lechermann, H. O. Jeschke, M. J. Rozenberg, R. Valentí, and A. F. Santander-Syro, Phys. Rev. B 94, 241110(R) (2016).

[61] http://chemistry-reference.com/standard\%20thermodynamic\% 20values.pdf, accessed: 2019-06-04.

[62] P. Zhang, P. Richard, T. Qian, Y.-M. Xu, X. Dai, and H. Ding, Rev. Sci. Instr. 82, 043712 (2011).

[63] A fit of the bare data yields an identical energy for the band bottom but a slightly lower effective mass $m^{*}=0.24 m_{e}$. In fact, the $k_{F}$ values derived from the momentum distribution curves of the raw data at the Fermi level are always smaller than the real ones because the Gaussian convolution with the experimental resolution shifts the band slightly towards $E_{F}$.

[64] P. Peercy and B. Morosin, Phys. Rev. B 7, 2779 (1973).

[65] A. Dieguez, A. Romano-Rodriguez, A. Vila, and J. Morante, J. Appl. Phys. 90, 1550 (2001).

[66] P. Sangeetha, V. Sasirekha, and V. Ramakrishnan, J. Raman Spectroscopy 42, 1634 (2011).

[67] C. Verdi, F. Caruso, and F. Giustino, Nat. Commun. 8, 15769 (2017). 